\title{
Are we close to bioengineering a human-sized, functional heart?
}

Doris A. Taylor, $\mathrm{PhD},{ }^{\mathrm{a}}$ Camila Hochman-Mendez, $\mathrm{PhD},{ }^{\mathrm{a}}$ and Luiz C. Sampaio, $\mathrm{MD}^{\mathrm{a}, \mathrm{b}}$

\footnotetext{
From ${ }^{\mathrm{a}}$ Regenerative Medicine Research and ${ }^{\mathrm{b}}$ Cullen Cardiovascular Surgery Research Laboratories, Texas Heart Institute, Houston, Tex.

Funded by Texas Heart Institute internal funds.

Received for publication May 2, 2019; revisions received June 7, 2019; accepted for publication June 16, 2019 available ahead of print Oct 24, 2019.

Address for reprints: Doris A. Taylor, PhD, Regenerative Medicine Research, Texas Heart Institute, PO Box 20345, Houston, TX 77225-0345 (E-mail: dtaylor@texasheart.org).

J Thorac Cardiovasc Surg 2020;159:1357-60

$0022-5223 / \$ 36.00$

Copyright (C) 2019 Published by Elsevier Inc. on behalf of The American Association for Thoracic Surgery https://doi.org/10.1016/j.jtcvs.2019.06.135
}

For 50 years, scientists have sought to build a replacement for a failing human heart. Initially, when the heart was viewed as only a pump, a mechanical device-such as the mechanical total artificial heart-was deemed a realistic strategy. However, as understanding of cardiovascular physiology has evolved, a biological approach has begun to seem feasible. Although total artificial heart technology is advancing, as exemplified by the BiVACOR (BiVACOR, Inc, Houston, Tex $)^{1}$ under extensive development at Texas Heart Institute, the contemporary mechanical total artificial heart still suffers from limitations, including large size, lack of long-term durability, externalized power source, lack of biocompatibility, and required lifetime anticoagulation therapy. $^{2,3}$ A bioengineered whole heart recellularized with patient-specific cells could theoretically overcome these limitations. However, building a biologic organ replacement is a much more complex undertaking than building a mechanical one; it requires multiple components, including vascularized scaffolds that mimic the structural complexity of the native heart; billions of human cardiac cells (cardiomyocytes, neurons, vascular cells) and a suitable mechanism for delivering them; a method of aligning cells within the scaffold to mimic both the structural and functional complexity of the organ; and a closed, sterile system that supports cardiac physiology over the weeks needed to mature the bioartificial structure. The vascularized scaffold problem was solved 10 years ago with the development of perfusion decellularization technologies, ${ }^{4}$ but scaffold recellularization has remained a challenge because of the massive numbers of cardiac cells needed to repopulate a human-sized organ and the difficulties of effectively transplanting the cells into the scaffold.

Recently, the possibility of building a transplantable heart from the recipient's own cells has stimulated interest in autologous transplantation. When human stem cell science was first applied to the cardiovascular system

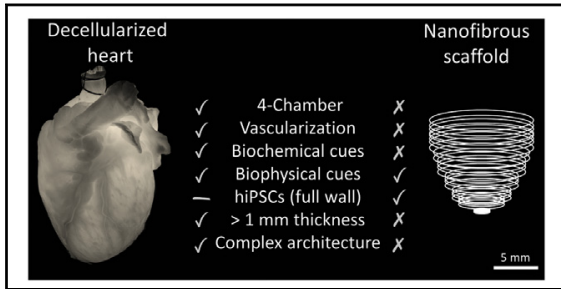

Comparison of decellularized and nanofiber-based heart scaffolds.

\section{Central Message}

Considering current efforts to bioengineer a (transplantable) heart, we examine 2 recent studies that incorporated iPSCs into scaffolds as either electrospun nanoscaffolds or 3Dprinted constructs.

This Invited Expert Opinion provides a perspective on the following paper: Nature Biomedical Engineering. 2018;2:930-941. https://doi.org/10.1038/s41551-018-0271-5.

See Commentary on page 1361.

20 years ago, ${ }^{5}$ scientists assumed that autologous transplantation would soon be possible. ${ }^{6}$ However, only now has human-induced pluripotent stem cell (hiPSC) technology matured to the point where this goal is truly feasible.

MacQueen and colleagues ${ }^{7}$ recently demonstrated noteworthy progress toward bioengineering a "functional heart" by describing the proper alignment and incorporation of hiPSCs into a single, ventricle-like, nanofiberbased scaffold. Furthermore, they were able to maintain the scaffold in a bioreactor that permitted quantification of physiologic function, thus demonstrating that hiPSC cardiomyocytes can be incorporated in at least in a small construct and reinforcing the potential for building a functional organ from the prospective recipient's own cells.

Although their work is a step forward, there are limitations to MacQueen and colleagues' approach. Their scaffold is only several cell layers thick, thinner than even a late fetal-stage human heart. With only a single, unvascularized chamber, the "myocardium" lacks the functional capability to support multichambered circulation of blood. Furthermore, critical cell types (eg, endothelial cells, cardiac fibroblasts, smooth muscle cells, nervous cells, pacemaker cells) are missing. 
Nonetheless, their sophisticated approach goes beyond an organ-on-a-chip ${ }^{8}$ and is an exciting proof of concept. Using the system, the investigators were able to demonstrate that when the ventricular construct was exposed to physiologic and pathologic pressure variations, consistent pressure-volume loops could be obtained. This advance introduces an additional method of measuring critical cardiovascular parameters, but it remains to be seen whether this small but complex approach will ultimately be useful in building a functional fourchambered organ.

More recently, scientists in Tel Aviv ${ }^{9}$ 3-dimensionally (3D) printed a dual-ventricle construct that more closely resembled a heart. Even so, it is far from being a translatable model, as cardiomyocyte/coronary microvasculature ratio was not contemplated and only large coronaries were printed.

Although these investigations are significant steps forward, they also illustrate critical limitations in the field. For example, MacQueen and colleagues have made an important advance by showing that hiPSC cardiomyocytes can be embedded in a scaffold and that the cells will align and contract, but they did not demonstrate that alignment and contraction can happen at the level of complexity or on the scale required for generating even a mouse heart, much less a human-size organ. Furthermore, although they are able to recellularize a ventricle-shaped scaffold, they cannot vascularize their scaffolds and thus are unable to generate a construct more than a few cells thick. Most recently, Wang and colleagues ${ }^{10}$ demonstrated the feasibility of 3D-bioprinting highly organized functional cardiac tissues with physiological and biomechanical properties similar to native cardiac tissue, but they were unable to demonstrate functionality of the construct.

\section{BUILDING COMPLEXITY}

It has been 10 years since the breakthrough publication of research demonstrating the feasibility of generating decellularized whole-heart scaffolds and repopulating small scaffolds $(\sim 1 \mathrm{~g})$ with rat neonatal cardiomyocytes. ${ }^{4}$ Since then, the scientific community has worked not only to scale up our model to human size but also to use cells from human lineages. ${ }^{11}$ In the quest for this tantalizing goal, investigators in the field have been able to bioengineer small but complex solid organ constructs (rat heart, rat liver lobe, rat lung lobe, cardiac patches, and small pieces of rat heart), and bioconstructs (rat heart, $<1-\mathrm{cm}$ patches), as well as large but simple ones (bladder dome, ${ }^{12}$ trachea ${ }^{13}$ ), but a "large and complex" prototype has yet to be developed. Although much progress has been made, the challenge of complete and appropriate recellularization-to build complex and vascularized cardiac tissue sufficient to support physiological function-has to date eluded scientists in the field. This is in part due to the limitations of generating cells and because successful delivery of both parenchymal and vascular cells to a complex organ remains challenging. This is especially true for a metabolically super-consuming organ such as heart, where proper cell perfusion and cell alignment are required for productive contraction to allow ejection of blood against the blood pressure, where decreased perfusion causes hibernation, and where misalignment promotes decreased ejection fraction and even organ failure.

The oxygen consumption and metabolic rate of cardiac tissue require a vascular bed extensive enough to provide nearly a one-to-one ratio of capillaries to cells. Beyond perfusion, the vascular tree provides access to growth factors and cyto/chemokines that modulate cell behavior. Equally critical is the extracellular matrix (ECM) framework that consists of an interstitium of structural proteins such as collagens and laminins, as well as proteoglycans and polysaccharides that bind growth factors and other chemokines and that align cells functionally. The efficient pumping action of the upper and lower chambers in fact requires intricate electrical and mechanical coordination provided in part by the ECM-all of which are missing from the current tissue-engineering constructs.

Today, science and technology have advanced to the point where one can envision the development of a bioartificial heart-manufactured for a specific patient and approved by the Food and Drug Administration-that could alleviate the shortage of transplantable organs, free patients from implanted mechanical devices, and reduce or eliminate the need for potentially toxic immunosuppressive drugs. An ideal tissue-engineered heart would be capable of in vitro remodeling, regeneration, and growth, and the need for antithrombogenic and immunosuppressive therapies would be reduced or eliminated. To realize this vision, the current technology must be scaled up and scaled out. Reproducing the complex structure and physiology de novo, even with modern 3D printing or electrospinning technology, is a formidable challenge.

In our laboratory, we remain focused on using the decellularized ECM of whole cadaveric organs as our scaffold of choice and resupplying human cells to this natural scaffold. ${ }^{14}$ Numerous synthetic substrates and biological materials have been studied as potential organ scaffolds, but none can provide the innate architectural complexity of the native heart. Decellularized ECM retains the micro- and macro-architecture and fullthickness vascularization of the native organ, biologically active molecules that modulate cell-cell and cell-ECM interactions, as well as structurally intact valves, chordae, and vasculature. ${ }^{15}$ Porcine hearts, which are strikingly similar to human hearts in size and anatomy, appear to provide an ideal scaffold for a bioartificial human heart. ${ }^{14}$ Furthermore, acellular porcine ECM is already approved for clinical use in multiple applications. ${ }^{16,17}$ In our hands, 
decellularized porcine ECM is acellular and retains $<9 \%$ of cadaveric levels of DNA.

Currently, the most critical obstacle to bringing this technology to clinical scale is the challenge of complete and appropriate recellularization. This entails repopulating the scaffold of choice with sufficient numbers of appropriate cells capable of organizing into a fully functional whole heart. The cellular bulk of the heart is formed by cardiomyocytes, endothelial cells, fibroblasts, smooth muscle cells, and specialized neural conduits, including pacemaker and Purkinje fibers. The 4 billion or so cardiomyocytes in a human heart are highly specialized and typically do not divide in vitro. But today, functional cardiomyocytes can be differentiated from induced pluripotent stem cells ${ }^{18}$ - human or animal—derived from readily available autologous cells such as skin, fibroblasts, or blood cells. Beyond cardiomyocytes, endothelial cells, which comprise about $40 \%$ of the cells of the heart, are critical for re-lining vessels, endocardium, and valves to prevent thrombosis. Furthermore, the use of autologous endothelial cells as a blood-facing barrier between parenchyma and blood flow can potentially overcome any $\alpha$-gal-related immunogenicity issues. Because a confluent bed of endothelial cells throughout the vascular tree and lining the endocardium and valves is probably necessary to mask the matrix, billions of endothelial cells are likely to be required for each human heart generated. We are addressing this hurdle by using large-scale automated cell factories that allow us to produce the billions of cells necessary to repopulate the scaffold. ${ }^{11}$ Yet, the cost to scale up/scale out production of these cells remains extremely high.

\section{FUTURE PERSPECTIVES}

The ultimate goal of cardiac tissue engineering is to build a bioartificial heart that could be approved by the Food and Drug Administration for transplant into patients with end-stage heart failure. Ideally, the bioconstruct would be capable of in vivo remodeling, regeneration, and growth and would reduce or eliminate the need for the postoperative immunosuppressive and antithrombogenic therapies necessitated by implantation of donor or mechanical organs. Furthermore, the transplantable hearts would be affordable and readily available, and the manufacturing process cost-effective and time-efficient. As we progress toward this goal, the developing technologies are now being used to develop test-beds for personalized drug testing. However, functional, human-sized organs have not yet been generated.

Research laboratories throughout the world are working to address the numerous challenges our primary goal presents. Small but complex tissue constructs such as the single-ventricle model developed by MacQueen and colleagues provide important tools to investigate many of the physiological and mechanical challenges facing investigative teams. Slightly more complex constructs such as those developed by Wang and colleagues are also critical to advancing the science. However, true breakthroughs will require repopulating a multichambered, tri-layered construct to produce muscle, nerves, and blood vessels and incorporating complex, specialized features such as valves.

The road forward will require not only advances in tissue engineering and cell biology but also devising the means to combine billions of human stem cells with vascularized, multichambered scaffolds, be they printed or garnered from cadaveric organs. These processes, in turn, will necessitate the development of next-generation bioreactors capable of replicating human cardiac physiology for the weeks needed to mature a recellularized scaffold and new approaches to cardiac transplant that allow organs to come online in a robust but protected fashion.

\section{Conflict of Interest Statement}

Dr Taylor holds a financial interest in Miromatrix Medical, Inc, and is entitled to sales royalty through the University of Minnesota for products related to the research described in this paper. This relationship has been reviewed and managed by the University of Minnesota and the Texas Heart Institute in accordance with its conflict of interest policies. This does not alter the author's adherence to Nature Biomedical Engineering's policies on sharing data and materials. All other authors have nothing to disclose with regard to commercial support.

\section{References}

1. Cohn WE, Timms DL, Frazier OH. Total artificial hearts: past, present, and future. Nat Rev Cardiol. 2015;12:609-17.

2. Copeland JG, Copeland H, Gustafson M, Mineburg N, Covington D, Smith RG, et al. Experience with more than 100 total artificial heart implants. J Thorac Cardiovasc Surg. 2012;143:727-34.

3. Levinson MM, Copeland JG, Smith RG, Cork RC, DeVries WC, Mays JB, et al. Indexes of hemolysis in human recipients of the Jarvik-7 total artificial heart: a cooperative report of fifteen patients. J Heart Transplant. 1986;5:236-48.

4. Ott HC, Matthiesen TS, Goh SK, Black LD, Kren SM, Netoff TI, et al. Perfusiondecellularized matrix: using nature's platform to engineer a bioartificial heart. Nat Med. 2008;14:213-21.

5. Rosen MR, Myerburg RJ, Francis DP, Cole GD, Marban E. Translating stem cell research to cardiac disease therapies: pitfalls and prospects for improvement. $J$ Am Coll Cardiol. 2014;64:922-37.

6. Taylor DA, Sampaio LC, Gobin A. Building new hearts: a review of trends in cardiac tissue engineering. Am J Transplant. 2014;14:2448-59.

7. MacQueen LA, Sheehy SP, Chantre CO, Zimmerman JF, Pasqualini FS, Liu X, et al. A tissue-engineered scale model of the heart ventricle. Nat Biomed Eng. 2018;2:930-41.

8. Conant G, Lai BFL, Lu RXZ, Korolj A, Wang EY, Radisic M. High-content assessment of cardiac function using heart-on-a-chip devices as drug screening model. Stem Cell Rev. 2017;13:335-46.

9. Noor N, Shapira A, Edri R, Gal I, Wertheim L, Dvir T. 3D printing of personalized thick and perfusable cardiac patches and hearts. Adv Sci (Weinh). 2019: 1900344.

10. Wang Z, Lee SJ, Cheng HJ, Yoo JJ, Atala A. 3D bioprinted functional and contractile cardiac tissue constructs. Acta Biomater. 2018;70:48-56.

11. Paccola Mesquita FC, Hochman-Mendez C, Morrissey J, Sampaio LC, Taylor DA. Laminin as a potent substrate for large-scale expansion of human 
induced pluripotent stem cells in a closed cell expansion System. Stem Cells Int. 2019;2019:9704945.

12. Atala A. Tissue engineering of human bladder. Br Med Bull. 2011;97:81-104.

13. Seguin A, Baccari S, Holder-Espinasse M, Bruneval P, Carpentier A, Taylor DA, et al. Tracheal regeneration: evidence of bone marrow mesenchymal stem cell involvement. J Thorac Cardiovasc Surg. 2013;145: 1297-304.e1292.

14. Taylor DA, Frazier OH, Elgalad A, Hochman-Mendez C, Sampaio LC. Building a total bioartificial heart: harnessing nature to overcome the current hurdles. Artif Organs. 2018;42:970-82.

15. Taylor DA, Sampaio LC, Ferdous Z, Gobin AS, Taite LJ. Decellularized matrices in regenerative medicine. Acta Biomater. 2018;74:74-89.
16. Ngo A, Hassager C, Thyregod HGH, Søndergaard L, Olsen PS, Steinbrüchel D, et al. Differences in left ventricular remodelling in patients with aortic stenosis treated with transcatheter aortic valve replacement with corevalve prostheses compared to surgery with porcine or bovine biological prostheses. Eur Heart J Cardiovasc Imaging. 2018; 19:39-46.

17. Petro CC, Prabhu AS, Liu L, Majumder A, Anderson JM, Rosen MJ. An in vivo analysis of Miromesh - a novel porcine liver prosthetic created by perfusion decellularization. J Surg Res. 2016;201:29-37.

18. Takahashi K, Yamanaka S. Induction of pluripotent stem cells from mouse embryonic and adult fibroblast cultures by defined factors. Cell. 2006;126: 663-76. 